\title{
Síndrome de Ovario Poliquístico: Una ventana a una gama de Alteraciones
}

Rubio-Esparza JJS, Medina-Aguiñaga D, Sánchez-López JL,Martínez-Muñoz FE, Valdivia-Esquivel $L R$, Terrones-Saldívar MC

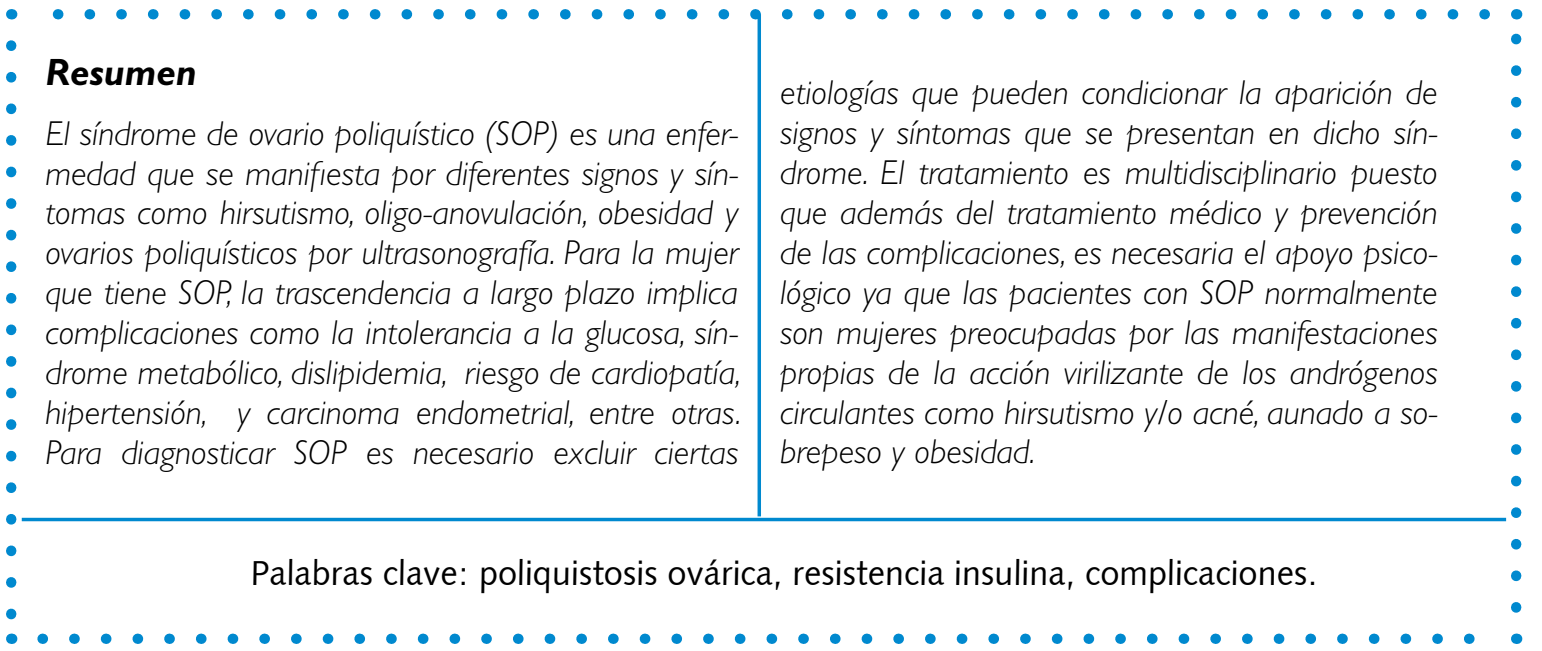

\section{Introducción}

El síndrome de ovario poliquístico (SOP) es una enfermedad que afecta entre el 5 y $10 \%$ de mujeres en edad reproductiva, con una influencia multifactorial en su etiología y una variable presentación clínica. ${ }^{1}$ Los síntomas y signos incluyen hirsutismo, obesidad, infertilidad, irregularidades menstruales, y anormalidades bioquímicas compatibles con hiperandrogenismo y resistencia a la insulina. ${ }^{1,2}$

La mayoría de las mujeres con SOP tienen niveles crónicamente elevados de la hormona luteinizante (LH) y la relación hormona luteinizante/hormona folículo estimulante (FSH) puede estar elevado, particularmente en mujeres con un índice de masa corporal $(I M C) \geq 30$; el IMC se correlaciona negativamente con la amplitud 
de pulsos de LH. La LH estimula a las células de la teca para producir andrógenos mediados por el CP450c17a, una enzima con actividad 17a-hidroxilasa y 17,20-liasa, la cual aumenta la producción de androstenediona. La androstenediona es convertida a testosterona por 17ß-hydroxiesteroide dehidrogenasa o aromatizada por la enzima aromatasa en las células de la granulosa para formar estrona. Esto permite que haya más testosterona libre circulante, que estimula los receptores androgénicos de la unidad pilosebácea lo cual lleva a los hallazgos clínicos de hirsutismo y acné. ${ }^{2-7}$

El exceso de andrógenos intraováricos produce el crecimiento de pequeños folículos sin desarrollo de un folículo dominante, lo cual condiciona una anovulación crónica que se manifiesta en la mayoría de los casos como oligo-amenorrea..$^{7-8}$

Se ha reportado que la resistencia a la insulina (RI) y la hiperinsulinemia se encuentra entre el 50 y el $70 \%$ en mujeres diagnosticadas de SOP, porcentaje significativamente más alta que en la población general, que es del 10 al $20 \%$, por lo que se les atribuye un papel importante en el síndrome ${ }^{5}$. Se han atribuido diversos mecanismos como causa de la RI en mujeres con SOP, algunos de los cuales se presumen intrínsecos al síndrome y otros relacionados con la adiposidad, dado que la obesidad es una de las manifestaciones clínicas asociadas más frecuentes. En estas mujeres, la acción transportadora de glucosa y la acción antilipolítica aparecen alteradas, aunque la unión al receptor permanece intacta. ${ }^{4}$ La hiperinsulinemia participa en el síndrome al reducir los valores de las proteínas transportadoras de andrógenos, incrementa la presencia de un factor de crecimiento similar a la insulina I (IGF-I) y estimula la producción androgénica ovárica. Se especula como causa de acantosis nigricans o hipertecosis ovárica, y finalmente, se relaciona la hiperinsulinemia con disfunción endotelial y dislipemia ${ }^{9}$ con disminución del colesterol HDL, triglicéridos elevados y pronunciada oxidación de $\mathrm{LDL}^{10}$. La resistencia a la insulina está exacerbada por la ganancia de peso, particularmente en la región abdominal, lo cual es común en pacientes con SOP ${ }^{11}$. La prevalencia de intolerancia a la glucosa y diabetes mellitus (DM) tipo 2 en mujeres con este síndrome es más alta, que la esperada para mujeres de edad similar ${ }^{12}$.

En 1990 los criterios para SOP definidos por la National Institutes of Health (NIH), eran, aumento de la morfología del ovario, ${ }^{1,4}$ además del hiperandrogenismo y/o hiperandrogenemia, oligo-anovulación, excluyendo otras etiologías como síndrome de Cushing, hiperprolactinemia, e hiperplasia adrenal congénita ${ }^{4}$. La definición revisada en el consenso de Rotterdam ESHRE/ASRM (European Society of Human Reproduction and Embryology/American Society for Reproductive Medicine) en el 2003 sobre SOP establece que para el diagnóstico de SOP se requieren 2 de los 3 criterios siguientes: oligo-anovulación, hiperandrogenemia y ovarios 
poliquísticos por ultrasonido. ${ }^{1,5,6} \mathrm{El}$ uso del criterio ultrasonográfico para definir el SOP emergió de igual manera en Rotterdam en el 2003. Los criterios por ultrasonido para diagnosticar SOP quedaron establecidos cuando se encuentra un aumento del volumen ovárico (volumen superior a $9 \mathrm{~cm} 3$ ), aunado a presencia de un número superior a 10 folículos entre 2-8 mm de diámetro, distribuidos en la superficie del ovario y aumento de la densidad del estroma ovárico. La distribución de los minúsculos quistes en un patrón periférico, la configuración en "cuerda de perlas", hiperecogenicidad estromal o volumen estromal incrementado son específicos para SOP. ${ }^{1,5}$ Aunque pueden estar ausentes las alteraciones ováricas por ultrasonido en el $20 \%$ de los casos de pacientes con SOP y por el contrario, se reportan en aproximadamente $15-20 \%$ de mujeres normales alteraciones ultrasonográficas ${ }^{5}$. Si un solo folículo presenta $10 \mathrm{~mm}$ de diámetro el escaneo debe ser repetido para calcular el volumen y el área. Un solo ovario poliquístico es suficiente para el diagnóstico. Sin embargo, hay que descartar otras causas de irregularidad menstrual e hiperandrogenismo, como: hiperplasia adrenal congénita no clásica, hiperprolactinemia, síndrome de Cushing, acromegalia y tumores adrenales/ováricos productores de andrógenos. ${ }^{12-13}$ En el cuadro 2 se muestra el diagnóstico diferencial de SOP4.

| | | | | | | | | | | | | | | | | | | | | | | | | | | | | | | | | | | | | | | | | | | | | | | | | | | | | | | | | | | | | | | | | | | | | | | | | | | | | | | | | | | | | | | | | | | | | | | | | | | | | | | | | | | | | | | | | | | | |

\section{Cuadro I}

\section{Criterios Diagnósticos del Síndrome de Ovarios Poliquísticos (SOPQ)}

Criterios de la conferencia de consenso de Rótterdam (2003) Se exigen 2 de los 3 criterios:

- Oligoovulación y/o anovulación

- Hiperandrogenismo clínico y/o de laboratorio

- Imagen ecográfica de OPQ

Más exclusión de las demás etiologías: hiperplasia suprearrenal congénita, tumores secretores de andrógenos y síndrome de Cushing

\section{Complicaciones}

\section{Síndrome metabólico}

El síndrome metabólico es mucho más común en mujeres con SOP que en la población femenina general de edad similar. Se ha reportado que en USA casi $50 \%$ de mujeres con SOP presentan síndrome metabólico. La conferencia de consenso de Rótterdam recomendó la detección del síndrome metabólico en las pacientes obesas con SOP. El síndrome metabólico se define por la presencia de al menos tres de los cinco criterios siguientes ${ }^{12}$

- Cintura $>88 \mathrm{~cm}$

- Triglicéridos $\geq 150 \mathrm{mg} / \mathrm{dl}$

- C-HDL $<50 \mathrm{mg} / \mathrm{dl}$

- Presión arterial (PA) sistólica $\geq 130$ o PA diastólica $\geq 85 \mathrm{~mm} \mathrm{Hg}$ 


\section{Cuadro 2}

\section{Condiciones de Exclusión en el Diagnóstico de Síndrome de Ovario Poliquístico}

\begin{tabular}{|c|c|c|c|c|}
\hline \multirow[t]{2}{*}{ Condición } & \multirow{2}{*}{$\begin{array}{c}\text { Hiperandrogenemia, } \\
\text { hiperandrogenismo } \\
\text { o ambos }\end{array}$} & \multirow{2}{*}{$\begin{array}{l}\text { Oligoamenorrea } \\
\text { o Amenorrea }\end{array}$} & \multicolumn{2}{|c|}{ Características distintivas } \\
\hline & & & Clínicas & $\begin{array}{l}\text { Hormonales } \\
\text { o bioquímicas }\end{array}$ \\
\hline $\begin{array}{l}\text { Hiperplasia } \\
\text { adrenal congénita } \\
\text { no clásica debida } \\
\text { a deficiencia de } \\
\text { 21-hidroxilasa }\end{array}$ & Sí & No es frecuente & $\begin{array}{l}\text { Historia familiar } \\
\text { de infertilidad, } \\
\text { hirsutismo, o } \\
\text { ambos; común } \\
\text { en judíos } \\
\text { Ashkenazi }\end{array}$ & $\begin{array}{l}\text { Nivel (basal) elevado } \\
\text { de 17-hidroxiprogesterona } \\
\text { en la mañana o } \\
\text { por estimulación }\end{array}$ \\
\hline Síndrome de Cushing & Sí & Sí & $\begin{array}{l}\text { Hipertensión, } \\
\text { estrías, } \\
\text { hematomas } \\
\end{array}$ & $\begin{array}{l}\text { Nivel elevado de } \\
\text { cortisol libre en orina } \\
\text { de } 24 \text { horas }\end{array}$ \\
\hline $\begin{array}{l}\text { Hiperprolactinemia } \\
\text { o prolactinoma }\end{array}$ & Ninguna o leve & Sí & Galactorrea & $\begin{array}{l}\text { Nivel elevado de } \\
\text { prolactina en plasma }\end{array}$ \\
\hline $\begin{array}{l}\text { Hipotiroidismo } \\
\text { primario }\end{array}$ & Ninguna o leve & $\begin{array}{l}\text { Puede estar } \\
\text { presente }\end{array}$ & $\begin{array}{l}\text { El bocio puede } \\
\text { estar presente }\end{array}$ & $\begin{array}{l}\text { Tirotropina elevada en } \\
\text { plasma y nivel } \\
\text { subnormal de tiroxina } \\
\text { en plasma; nivel de } \\
\text { prolactina puede estar } \\
\text { incrementado }\end{array}$ \\
\hline Acromegalia & Ninguna o leve & Frecuente & $\begin{array}{l}\text { Alargamiento } \\
\text { acral, } \\
\text { características } \\
\text { toscas, } \\
\text { prognatismo } \\
\end{array}$ & $\begin{array}{l}\text { Factor de crecimiento } \\
\text { insulin-like I } \\
\text { incrementado en } \\
\text { plasma }\end{array}$ \\
\hline $\begin{array}{l}\text { Falla ovárica } \\
\text { prematura }\end{array}$ & Ninguna & Sí & $\begin{array}{l}\text { Puede estar } \\
\text { asociada con } \\
\text { otras } \\
\text { endocrinopatías } \\
\text { autoinmunes } \\
\end{array}$ & $\begin{array}{l}\text { FSH elevada en } \\
\text { plasma o nivel } \\
\text { subnormal de } \\
\text { estradiol. }\end{array}$ \\
\hline Obesidad simple & Frecuente & No es frecuente & $\begin{array}{l}\text { Diagnosticada } \\
\text { por exclusión }\end{array}$ & Ninguna \\
\hline $\begin{array}{l}\text { Virilización } \\
\text { adrenal o } \\
\text { neoplasia ovárica }\end{array}$ & Sí & Sí & $\begin{array}{l}\text { Clitoromegalia, } \\
\text { hirsutismo } \\
\text { extremo, o } \\
\text { alopecia en } \\
\text { patrón } \\
\text { masculino } \\
\end{array}$ & $\begin{array}{l}\text { Nivel extremadamente } \\
\text { elevado de } \\
\text { andrógenos en } \\
\text { plasma }\end{array}$ \\
\hline $\begin{array}{l}\text { Condición } \\
\text { relacionada a } \\
\text { fármacos* }^{*}\end{array}$ & Frecuente & Variable & $\begin{array}{l}\text { Evidencia } \\
\text { proveída por } \\
\text { historia }\end{array}$ & Ninguna \\
\hline
\end{tabular}

*Una condición relacionada a fármacos es debida al uso de andrógenos, ácido valproico, ciclosporina u otros fármacos. 
- Intolerancia a los carbohidratos: 1,10 $\mathrm{g} / \mathrm{l}<$ glucemia en ayunas $<1,26 \mathrm{~g} / \mathrm{l}$, y/o: 1,40 g/l < glucemia a las 2 horas de una hiperglucemia por sobrecarga oral de glucosa (HSOG) de <1,99 g/l. (HSOG: hiperglucemia por sobrecarga oral de glucosa.)

Pacientes con fenotipo leve de SOP tienen una baja prevalencia de síndrome metabólico pero, en estas pacientes también, el síndrome metabólico es dos veces más frecuente que en la población normal. Todas las mujeres obesas o con sobrepeso con SOP deben estudiarse para síndrome metabólico, cuando el síndrome no se encuentra, el estudio debe repetirse cada 2-3 años ${ }^{13}$.

\section{Dislipidemia}

La dislipidemia característica de la resistencia a la insulina y rangos desfavorables de lipoproteínas proaterogénicas están presentes en mujeres con SOP ${ }^{14}$. Así mismo, los factores de riesgo para enfermedad cardiovascular (obesidad, hipertensión arterial, intolerancia a la glucosa o DM 2, hiperinsulinemia, dislipidemia y alteraciones de la coagulación), están asociados a un riesgo incrementado de desarrollar ateroesclerosis. La ateroesclerosis es un proceso sistémico caracterizado por inflamación crónica que afecta todos los territorios vasculares y causa cambios en su estructura y función; estos cambios culminan en trombosis arterial ${ }^{15}$.

\section{Complicaciones obstétricas}

La insulinoresistencia del SOP, puede predisponer a la diabetes mellitus gestacional, la mujer embarazada que desarrolla hipertensión generalmente muestra una disminución de la sensibilidad a la insulina. En un estudio se reportaron una tendencia a valores mayores de presión arterial en las pacientes con SOP en el tercer trimestre y durante el parto ${ }^{16-19}$. El objetivo del estudio fue determinar la incidencia de hipertensión, preeclampsia y diabetes en un grupo de embarazadas con antecedentes de SOP reconocidas previa al embarazo. Cincuenta pacientes embarazadas con antecedentes de SOP se incluyeron en el estudio. El grupo control consistió en 500 mujeres con gestación normal. Comparado con el grupo control, en los casos de SOP se encontró mayor índice de masa de corporal, de hipertensión crónica ( $p=0,05)$, mayor incidencia de parto prematuro y complicaciones neonatales $(p=0,05)$ y mayor incidencia de diabetes, especialmente en el grupo insulino resistente $(p=0,05)$. Se concluye que la frecuencia de hipertensión crónica y la diabetes gestacional es significativamente mayor en embarazadas con antecedentes de SOP comparado con los controles $^{20}$.

\section{Impacto sobre la Fertilidad}

El daño de la función reproductiva en la mujer con SOP está usualmente explicado por anovulación. ]El SOP es considerado como la causa más común de infertilidad anovulatoria, explicando más del $70 \%$ de los casos. Sin embargo, la falla en la implantación y la tendencia a una incidencia más alta de aborto, indica que factores endometriales pueden ser también de importancia para la disfunción reproductiva en SOP. Existe un incremento en evidencia de que las anormalidades endocrinas y metabólicas, tales como la no oposición a los estrógenos, hiperandrogenismo e hiperinsulinemia en SOP, pueden tener efectos adversos sobre el endometrio y contribuir a la infertilidad en estas pacientes. Aún más, el hiperandrogenismo ovárico se ha asociado con reducida calidad de ovocitos u embrión ${ }^{21}$.

\section{Carcinoma endometrial}

La evidencia de un riesgo incrementado de carcinoma endometrial en SOP es incompleta y contradictoria. Intuitivamente pa- 
rece como que la mujer con el síndrome quien tiene periodos irregulares o amenorrea tiene mayor riesgo por causa de los efectos estimulantes estrogénicos sin oposición. Existe evidencia que las mujeres con infertilidad anovulatoria tienen riesgo de desarrollar hiperplasia endometrial, y en algunos casos esta será atípica y potencialmente maligna. Este es también el caso de SOP que es la causa más común de anovulación, pero estos hechos no deben combinarse para concluir que estas mujeres individuales con SOP necesariamente tendrán alto riesgo de desarrollar cáncer endometrial. Es más común que este riesgo aplique sólo a un subgrupo de mujeres, posiblemente aquellas quienes son también obesas ${ }^{22}$.

\section{Enfermedad fibroquística mamaria}

La enfermedad mamaria fibroquística se describe como cambios benignos comunes que involucran los tejidos de la mama. En un estudio realizado a 53 mujeres con SOP y a un grupo control de 40 mujeres a las que se les realizó ultrasonografía de mama se encontró una asociación estadísticamente significativa entre las pacientes con SOP y la enfermedad mamaria fibroquística; por lo tanto, es recomendable que las mujeres con SOP deban ser evaluadas en busca de mastopatía fibroquística ${ }^{23}$.

\section{Apnea del sueño}

La prevalencia de este síndrome en las mujeres con SOP es mayor y no puede explicarse sólo por la obesidad ${ }^{24,25}$. Parece que la resistencia a la insulina es un mejor factor predictivo del síndrome de apnea del sueño que la edad, el IMC o las concentraciones plasmáticas de testosterona ${ }^{24}$.

\section{Tratamiento}

Uno de los objetivos del tratamiento del SOP es disminuir la prevalencia de las complicaciones reproductivas, cutáneas, metabólicas y psicológicas.

\section{Alteraciones del ciclo menstrual}

La anovulación crónica incrementa el riesgo de hiperplasia y de carcinoma endometriales, la administración secuencial de progestágenos durante la teórica fase luteínica o de un tratamiento combinado de estrógenos y progestágenos permite inhibir la proliferación endometrial. Sin embargo, el uso de los anticonceptivos es discutible en las pacientes con SOP, ya que pueden aumentar el riesgo de anomalías metabólicas que favorecen la resistencia a la insulina, la intolerancia la glucosa y las dislipidemias, así como el riesgo de trombosis ${ }^{27}$. Para aquellas mujeres con irregularidades menstruales y características clínicas de hiperandrogenismo, la administración de acetato de ciproterona ( $2 \mathrm{mg}$ ) y etinilestradiol (35 $\mu \mathrm{g}$ ) ayuda a regularizar el sangrado, y disminuye el acné y/o hirsutismo, además provee anticoncepción ${ }^{28}$.

\section{Infertilidad}

La causa de infertilidad en pacientes con SOP es generalmente una disminución de la ovulación por falla folicular. La mayoría de los ciclos son anovulatorios y la inducción de la ovulación es esencial. El citrato de clomifeno es un antagonista oral de los estrógenos que eleva los niveles circulantes de FSH e induce crecimiento folicular. El régimen inicial es de $25-50 \mathrm{mg} /$ día por 5 días. Deben monitorearse los niveles de estrógenos, realizar revisión ultrasonográfica folicular y niveles de progesterona ${ }^{13-28}$. Aunque existe la combinación de citrato de clomifeno con metformina, en pacientes con SOP e infertilidad anovulatoria, sin tratamiento previo, la administración de metformina en combinación con citrato de clomifeno no ha demostrado ser mejor que la monoterapia (metformina sola o citrato de clomifeno solo) ${ }^{29}$

\section{Hirsutismo y acné}

La drospirenona es un progestágeno análogo a la espironolactona con actividad an- 
tiandrogénica y antimineralocorticoidea. La utilización de anticonceptivos combinados de etinilestradiol y drospirenona, mejora el hirsutismo y protege al endometrio aunque se le cuestiona un potencial efecto sobre la resistencia a la insulina, la intolerancia a la glucosa, la reactividad vascular y la coagulabilidad.

El acetato de ciproterona inhibe de manera competitiva la unión de la testosterona y de la dihidrotestosterona (DHT) al receptor de andrógenos. La espironolactona, en dosis de 100 a 200 mg por día, posee un moderado efecto antiandrogénico y pareciera actuar de manera sinérgica a los anticonceptivos orales.

La flutamida es un potente antiandrógeno no esteroide y es efectivo contra el hirsutismo, aunque puede producir disfunción hepática, por lo que su uso es limitado ${ }^{30}$.

\section{Resistencia a la insulina}

Antes de iniciar un tratamiento farmacológico para mejorar la resistencia a la insulina, debe recomendarse a la paciente la disminución de peso, aunada a una menor ingesta de carbohidratos. Existen datos publicados que sugieren que una dieta con baja carga glicémica en combinación con medicamento puede contribuir al mejoramiento de los síntomas en la paciente con SOP ${ }^{11-31}$.La American Association of Clinical Endocrinologists recomienda que la metformina sea considerada la intervención inicial en la mayoría de las mujeres con síndrome de ovario poliquístico, particularmente en aquellas quienes tienen sobrepeso $u$ obesidad ${ }^{31}$.

Las recomendaciones para el manejo de la paciente con SOP se muestran en el cuadro $3^{26}$.

\section{Cuadro 3}

Recomendaciones para el manejo de la mujer con SOP.

\begin{tabular}{|l|l|}
\hline Regular ciclos menstruales & $\begin{array}{l}\text { Prescribir hormonales combinadas, el progestágeno se preferencia } \\
\text { con actividad antiandrogénica (drospirenona). En caso de buscar un } \\
\text { embarazo dar ciclos de inducción de ovulación, y metformina. } \\
\text { Considerar ténicas de reproducción asistida }\end{array}$ \\
\hline Metabólicas & $\begin{array}{l}\text { Valorar riesgo de diabetes y enfermedad cardiovascular } \\
\text { Fomentar reducción de peso } \\
\\
\text { Promover y fomentar terapias de estilo de vida, } \\
\text { sobre como nutrición y actividad física. }\end{array}$ \\
\hline Psicosocial & Reforzar autoestima \\
& Identificar tendencia a la depresión \\
& Discutir manejo de estrés \\
\hline Cosmético & Utilización de hormonales con actividad antiandrogénica \\
& Considerar espironolactona $50-100$ mg \\
& Considerar terapia láser, y electrolisis \\
\hline Apnea del Sueño & Revisión para apnea del sueño \\
& Referir para estudio del sueño si está indicado \\
\hline
\end{tabular}




\section{Conclusiones}

El síndrome de ovario poliquístico es una patología compuesta por una florida gama de signos y síntomas, la cual, debe ser estudiada de forma concienzuda y analítica; basándose en descartar otras patologías similares. No obstante, el rol del médico no termina al resolver las alteraciones menstruales o la oligo-anovulación ni las manifestaciones propias del hirsutismo; sino que debe mantenerse una vigilancia estrecha de la paciente por los riesgos inherentes a largo plazo, de desarrollar diabetes mellitus, carcinoma endometrial, afecciones cardiovasculares e hipertensión. Dichas afecciones han sido demostradas en múltiples estudios publicados recientemente. Además, de la variedad de tratamientos existentes para el SOP será necesario hacer un balance con los deseos de maternidad de la paciente, respetando su voluntad.

\section{Bibliografía}

1 R.F. O'Brien, MD and S.J. Emans, MD. Polycystic Ovary Syndrome in Adolescents. J Pediatr Adolesc Gynecol (2008) 21:119e128

2 Lee Radosh, MD. Drug Treatments for Polycystic Ovary Syndrome. Am Fam Physician. 2009; 79(8):671-676.

3 Tracy L. Setji, MD, Ann J. Brown, MD, MHS. Polycystic Ovary Syndrome: Diagnosis and Treatment. The American Journal of Medicine (2007) 120, 128-132

4 David A. Ehrmann, M.D.Polycystic ovary syndrome medical progress. N Engl J Med 2005; 352:122336.

5 R Tur, N Parera, J Ybarra, PN Barrl. Ovarios poliquísticos: actualizació. Encycl Méd Chir (Editions Scientifiques et Médicales Elsevier SAS, Paris, tous droits réservés), Gynécologie, 133-A-10, 2002, $4 p$

6 Shroff R, Kerchner A, Maifeld M, Van Beek J.R. E, Jagasia D, Dokras A. Young Obese Women with Polycystic Ovary Syndrome Have Evidence of Early Coronary Atherosclerosis. J Clin Endocrinol Metab, December 2007, 92(12):4609-4614

7 Walter Futterweit, MD, FACP, FACE. Polycystic Ovary Syndrome: A Common Reproductive and Metabolic Disorder Necessitating Early Recognition and Treatment. Prim Care Clin Office Pract 34 (2007) 761-789

8 Puurunen J, Piltonen T, Jaakkola P, Ruokonen A, Morin-Papunen L, Tapanainen S. J. Adrenal Androgen Production Capacity Remains Highup to Menopause in Women with Polycystic Ovary Syndrome. J Clin Endocrinol Metab, June 2009, 94(6):1973-1978.

9 F Pazos, A Rodríguez-Caballero. Síndrome del ovario poliquístico: nuevas perspectivas. Endocrinol Nutr. 2004; 51:506-11.

10 Macut D, Panidis D, Glis `ic' B, Spanos N, Petakov M, Bjekic' J, Stanojlovic' O, Rousso D, Kourtis A, Boz 'ic' I, Damjanovic S. Lipid and lipoprotein profile in women with polycystic ovary syndrome. Can. J. Physiol. Pharmacol. 86: 199-204 (2008)
11 Herriot A. M, Whitcroft S, Jeanes Y. An retrospective audit of patients with polycystic ovary syndrome: the effects of a reduced glycaemic load diet. J Hum Nutr Diet, 21, pp. 337-345 (2008)

12 Gagnon C, Baillargeon J.P. Suitability of recommended limits for fasting glucose tests in women with polycystic ovary syndrome. CMAJ. March 27, 2007, 176(6): 933-937

13 Marcin Stankiewicz, Robert Norman. Diagnosis and Management of Polycystic Ovary Syndrome. A Practical Guide. Drugs, 2006; 66 (7):903-912

14 Giallauria F, Palomba S, Maresca L, Vuolo L, Tafuri D, Lombardi G, Colao A, Vigorito C, Orio F. Exercise training improves autonomic function and inflammatory pattern in women with polycystic ovary syndrome (PCOS). Clinical Endocrinology (2008) 69, 792 798.

15 Soares M. F, Vieira S. C, Martins P. W, Franceschini S. A, dos Reis R. M, Silva de Sa' and Rui Alberto Ferriani M. F. Increased arterial stiffness in nonobese women withpolycystic ovary syndrome (PCOS) without comorbidities: one more characteristic inherent to the syndrome? Clinical Endocrinology, 71, 406-411(2009).

16 Bjercke S, Dale $P$, Tanbo T et al. 2002. Impact of insulin resistance on pregnancy complications and outcome in women with polycystic ovary syndrome. Gynecol Obstet Invest 54: 94-98.

17 Sánchez I, Azziz R. 2000. Síndrome de ovario poliquístico, evaluación, diagnóstico, tratamiento y pronóstico. Rev Obstet Ginecol Venez 60: 47-57.

18 Szymanska $M$, Suchonska B, Bobrowska $K$ et al. 2003. Disorders of glucose tolerance among women with pregnancy induced hypertension. Ginekol Pol 74: 1450-1455.

19 Dodd J, Crowther C, Antoniou G et al. 2007. Screening for gestational diabetes: the effect of varying blood glucose definitions in the prediction of adverse maternal and infant health outcomes. Aust NZ J Obstet Gynaecol 47: 307-312.

20 Santos-Bolivar et al. Complicaciones obstétricas y 
ovarios poliquísticos. MedULA 17: 107-110, 2008.

21 Hirschberg A. L. Polycystic Ovary Syndrome, Obesity and Reproductive Implications. Women's Health, Volume 5, Number 5, September 2009, pp. 485496.

22 Hardiman P, Pillay S. O, Atiomo W. Polycystic ovary syndrome and endometrial carcinoma. Lancet 2003; 361: 1810-12

23 Gumus I. I, Koktener A, Dogan D, Turhan O. N. Polycystic ovary syndrome and Wbrocystic breast disease: is there any association? Arch Gynecol Obstet (2009) 280:249-253

24 Vgontzas AN, Legro RS, Bixler EO, Grayev A, Kales A, Chrousos GP. Polycystic ovary syndrome is associated with obstructive sleep apnea and daytime sleepiness: role of insulin resistance. J Clin Endocrinol Metab 2001; 86:517-20.

25 Gopal M, Duntley S, Uhles M,Attarian H. The role of obesity in the increased prevalence of obstructive sleep apnea syndrome in patients with polycystic ovary syndrome. Sleep Med 2002; 3:401-4.

26 Setji TL, Brown AJ. Polycystic ovary syndrome and type 2 diabetes. In: Feinglos MN, Bethel MA, eds.
Type 2 Diabetes Mellitus: An Evidence-Based Approach to Practical Management. Totowa, New Jersey: Humana Press; 2006:in press.

27 L. Théron-Gérard, I. Cédrin-Durnerin, J.-N. Hugues. Recomendaciones para el diagnóstico y el seguimiento del síndrome de ovarios poliquísticos. EMC (Elsevier Masson SAS, Paris), Gynécologie, 145-A10, 2008.

28 Bhathena R. K. Therapeutic options in the polycystic ovary syndrome. J Obst and Gynae, February 2007; 27(2): $123-129$

29 Palomba S, Pasquali R, Orio Jr F, Nestler E. J, Clomiphene citrate, metformin or both as first-step approachin treating anovulatory infertility in patients with polycysticovary syndrome (PCOS): a systematic review of head-to-head randomized controlled studies and meta-analysis. Clinical Endocrinology (2009) 70, 311-321

30 Discacciati V. Síndrome de ovario poliquístico. Evid. actual. práct. ambul; 10(6):178-180, Novdic.(2007).

31 Nestler E. J, M.D. Metformin for the Treatment of the Polycystic Ovary Syndrome. N Engl J Med 2008; 358:47-54.

\section{LUXMÍDICA}

\section{Normas para la publicación de artículos en lla revista}

La Revista LUX MÉDICA publica artículos de interés en el área de la salud en inglés o en español, y como criterios generales de publicación se tomará en cuenta la solidez científica del trabajo, la originalidad, actualidad y oportunidad de la información. Los trabajos deberán ser originales e inéditos. De ser aceptados por el Consejo Editorial, la revista LUX MÉDICA se reserva todos los créditos de publicación.

\section{Clasificación de artículos y secciones de la revista}

Artículos originales: Son informes de investigaciones relacionadas al campo de la medicina y salud pública, deberán ser inéditos y elaborados de acuerdo a las normas mencionadas. Contendrá hasta 1500 palabras, 20 a 30 referencias y cuadros, más figuras en número igual a la mitad menos uno de página de texto a doble espacio (4 cuadros más figuras para 10 páginas). Pueden ser informes de nuevos síndromes o enfermedades, de aspectos etiológicos, experiencias clínicas, ensayos terapéuticos, investigación epidemiológica o metodológica y de estudios básicos con repercusión en la clínica.

Actualizaciones o revisiones: Se refiere al análisis más completo posible y actualizado sobre un tema en el campo de la medicina; estará integrado hasta por seis mil palabras. Deberá ser un enjuiciamiento detallado y crítico de experiencias relevantes en la medicina, de preferencia de tópicos básicos o clínicos.

Caso clínico: Son artículos de presentación de casos aislados o grupos poco numerosos de pacientes, con patologías diferentes o poco comunes. Su extensión es de hasta 750 palabras, 10 referencias y 2 tablas mas figuras. Puede ampliarse si involucra a más de un solo caso.

Evidencia en imágenes: Fotografías de imágenes clínicas, piezas quirúrgicas, estudios de gabinete etc., relevantes por su originalidad o extrańeza, presentadas junto con un breve resumen y propuesta didáctica. La mejor imagen será incluida en la portada de la revista a manera de acertijo.

Desde las aulas: Espacio dirigido a los alumnos de las diferentes carreras biomédicas, tanto de pregrado como de postgrado, quienes deberán acompañar su trabajo con la supervisión y/o asesoría de cualquier médico acreditado. De preferencia se aceptarán revisiones bibliográficas o proyectos de investigación de incidencia local.

Ars médica: Espacio dedicado a escritores y artistas miembros o no de la comunidad médica, quienes podrán aportar textos y obras artísticas que contribuyan a mejorar la cultura en salud de la comunidad.

El formato diferente y su cualidad de dossier desprendible tiene por objeto su amplia difusión más allá del área del interés estrictamente médico. 